\title{
A Brief Evidence-based Intervention to Enhance Workplace Well-being and Flourishing in Health Care Professionals: Feasibility and Pilot Outcomes
}

\author{
Abbie O. Beacham, $\mathrm{PhD}^{1 *}$, Norah Janosy, $\mathrm{MD}^{2,3}$, Alison Brainard, $\mathrm{MD}^{2}$, Jennifer Reese, $\mathrm{MD}^{2,3}$
}

\begin{abstract}
Introduction: The deleterious direct and indirect effects of burnout among physicians and other health care professionals and learners have been well documented. Recently, there have been calls to shift the focus from distress and burnout to enhancing well-being of health care providers and learners. To this end, we developed a brief, well-being focused intervention entitled "CU Flourish" embedded in PERMA theory: positive emotion $(\mathrm{P})$, engagement $(\mathrm{E})$, relationships $(\mathrm{R})$, meaning $(\mathrm{M})$, and accomplishment $(\mathrm{A})$ with a sixth domain - Health in our working model. Within this framework, elements of values-based interventions, mindful awareness and psychological flexibility inherent to Acceptance and Commitment Therapy were integrated into the appropriate PERMAH domain modules. In this pilot study we examined the effectiveness of CU Flourish in increasing scores of workplace Well-being and Flourishing in a sample of health care professionals and learners.
\end{abstract}

Methods: Participants $(\mathrm{N}=20)$ completed pre-post surveys including validated measures and open-ended questions as part of the program evaluation.

Results: At post-course measurement, participants' overall PERMAH Workplace Well-being scores, Flourishing Ratio, and Mindfulness scores increased.

Conclusion: Overall, the CU Flourish curriculum may be an effective and desirable intervention for enhancing workplace well-being among health care professionals and learners.
DOI: 10.18297/jwellness/vol2/iss1/7 Received Date: January 24, 2020 Accepted Date: February 19, 2020 Publication Date: April 10, 2020

Website: https://ir.library.louisville.edu/ jwellness/

Recommended Citation: Beacham, Abbie O.; Janosy, Norah; Brainard, Alison; Reese, Jennifer (2020) "A brief evidence-based intervention to enhance Workplace Well-being and Flourishing in health care professionals: Feasibility and pilot outcomes" Journal of Wellness: Vol. 2 : Iss. 1, Article 7.

Affiliations:

University of Louisville School of Dentistry, ${ }^{2}$ University of Colorado School of Medicine ${ }^{3}$ Children's Hospital Colorado

\section{INTRODUCTION}

The deleterious direct and indirect effects of burnout among physicians and other health care professionals and learners have been well documented [1-3]. Levels of burnout are quite variable in different settings and among different specialties and professions [4] but reach levels to be regarded as constituting a public health crisis. Certainly, it is clear that "... demands on all physicians [and health care providers] have never been higher and ... There's the personal pressure of appearing infallible to patients, and the stigma attached to asking for help" [4].

The factors that contribute to burnout may be conceptualized as falling into three categories: 1) System level (including job-specific resources and demands) 2) Team level (including interaction among team members/colleagues) and 3) Individual resources. It has been asserted that burnout is not an "individual problem triggered by personal limitations" and that efforts spent on mitigation of burnout effects would best be placed on system level factors [3]. Slavin et al [5] suggest that systems and programs that primarily focus on "treating distressed individuals and fail to look at the workplace environment" fall short of addressing the full extent and complexity of the problem. We suggest that the answer lies in multifaceted, inclusive and proactive approaches across all levels

*Correspondence To: Abbie O. Beacham, Ph.D.

Email: Abbie.Beacham@louisville.edu

\section{JOURNAL}

of the system, team and individual resources to address this complex problem.

In light of all that has been gleaned regarding how burnout might best be addressed, there have been calls to shift the focus from distress and burnout to enhancing well-being of health care providers and learners. It has been further underscored that health care professional wellness should not be defined solely by the absence of or decrease in levels of burnout [6]. Rather Eckleberry-Hunt and colleagues [6] suggest a paradigm shift toward primary and secondary prevention to enhance levels of overall wellness, a focus on strength building (as opposed to weaknesses or pathology) and, ultimately, actively promote impactful culture change.

Some programs and interventions developed to address this need have focused on fostering increased levels of resilience - developing the ability to bounce back after adversity. We, however, consider a shift beyond the lowest rebound criterion of resilience toward Flourishing [7] to be the optimal target in assisting individuals in a process of growth, flexibility and overall well-being.

To this end, we developed a brief well-being focused intervention entitled "CU Flourish" embedded in the five domains defined by Seligman's [7] PERMA theory: positive emotion (P), engagement (E), relationships (R), 
meaning $(\mathrm{M})$, and accomplishment $(\mathrm{A})$. In accordance with Butler and Kern [8] a sixth domain - Health - was added to our working model. Within this framework, elements of values-based interventions, mindful awareness and psychological flexibility inherent to Acceptance and Commitment Therapy $[9,10]$ were integrated into the appropriate PERMAH domain modules. Additionally, the Broaden-and-Build Theory of Positive Emotions [10] further informed didactic and experiential components of the intervention.

The CU Flourish curriculum is brief, evidence-based, accessible, and can be applicable to a broad range of recipients. In guiding the selection of content, we adopted the definition of evidence-based practice as the integration of best research evidence with clinical expertise and consumer values as a guide $[11,12]$.

Topics covered in the CU Flourish curriculum are designed to be presented in 45 to 60 minute group classes over five sessions. However, in order to facilitate a four-class format, content from one module was distributed and combined with other content. The CU Flourish curriculum is considered "universal" such that the information and exercises can be understood and meaningfully tailored and applied to persons of various backgrounds, age cohorts and professions.

The evidence in support of the efficacy and effectiveness of positive psychology interventions (PPIs) is robust. Exercises included in three of the five CU Flourish modules are based on and adapted from widely utilized PPIs. Studies employing the PPI content and interventions have been associated with increased subjective and psychological well-being and decreased distress with effect sizes ranging from small to large and have been viewed as being largely sustainable. For a more comprehensive review of PPIs the reader is directed to Bolier et al [13]. The concepts from the Broaden-and-Build [10] and affective neuroscience integrate the role of affective plasticity [14] into the content and rationale for PPIs in the modules. Finally, practical mindfulness and present moment experience exercises as well as elements of Acceptance and Commitment Therapy (i.e., values clarification, decision making and congruence; [15]) are woven into the curriculum. All components of CU Flourish are organized within the overarching PERMAH model framework (Table 1).

The present study reports initial findings of a pilot program development intervention. Among our primary outcomes, we predicted increases in overall and individual workplace Well-being scores [8] and Flourishing positivity ratios [16] from pre- to post-course. We also predicted that our secondary outcomes Resilience [17] and Mindfulness [18] scores would increase from pre- to post-course. Finally, we aimed to evaluate survey perceptions of the acceptability and feasibility of CU Flourish for purposes of more widespread dissemination.

Table 1: CU Flourish Overview of Intervention Content

\begin{tabular}{|c|c|}
\hline Module & $\underline{\text { Session Content }}$ \\
\hline Introduction to Total Well-Being & $\begin{array}{l}\text { Didactic: Rethinking Work-Life Balance, } \\
\text { PERMAH Well-being model components } \\
\text { Experiential: "A time you were at your best"7; Brief } \\
\text { present moment awareness exercise, Adapted form of } \\
\text { PERMAH workplace profiler } \\
\text { PERMAH Domain: P, R }\end{array}$ \\
\hline Pragmatic Mindfulness & $\begin{array}{l}\text { Didactic: Scientific underpinnings of mindfulness, } \\
\text { Definition and application of mindfulness in daily life } \\
\text { Experiential: Brief introduction to diaphragmatic } \\
\text { breathing, posture and brief mindful exercises for daily } \\
\text { application, Self-assessment of mindfulness } \\
\text { PERMAH Domain: E }\end{array}$ \\
\hline Finding your Values Compass & $\begin{array}{l}\text { Didactic: Defining and assessing values (versus goals) } \\
\text { Experiential: Values "Bulls Eye" exercise }{ }^{22} \text {,"Choice } \\
\text { Point"15 applied to activity decision making } \\
\text { PERMAH Domain: M, A }\end{array}$ \\
\hline $\begin{array}{l}\text { NOT Pollyanna: Positive and Negative } \\
\text { Emotion in Daily Life }\end{array}$ & $\begin{array}{l}\text { Didactic: Role of positive and negative emotion in } \\
\text { everyday life, application of neuroplasticity and habitual } \\
\text { responding and cultivating positive emotion } \\
\text { Experiential: Video and " } 3 \text { Good Things"7 } \\
\text { PERMAH domain: } \mathrm{P}, \mathrm{R}\end{array}$ \\
\hline True(er) Grit & $\begin{array}{l}\text { Didactic: Definition and applications of concept of } \\
\text { Grit }^{23} \text {, Growth Mindset }{ }^{24} \text { and application of types of } \\
\text { Grit } \\
\text { Experiential: Video clips examples, Curiosity } \\
\text { Assessment }^{10} \\
\text { PERMAH Domain: A }\end{array}$ \\
\hline
\end{tabular}




\section{METHODS}

\section{Procedures}

The course (i.e., intervention) was offered as a part of a Resilience Program that served faculty, learners and staff in a School of Medicine at a large academic health sciences center. Participants registered online for the $\mathrm{CU}$ Flourish course via a campus wide email invitation. As part of the course, participants were invited to complete a pre- and post-course online survey anonymously with a self-selected fictitious name. Participants completed surveys 1 - week prior and within 1-week after they attended a series of four classes covering five topic modules (see Table 1). The course was instructed by a licensed psychologist with extensive experience in the development and teaching of the course content.

\section{Participants}

Among participants $(\mathrm{N}=26)$ in two cohorts who attended the course sessions (Cohort $1 \mathrm{n}=12$; Cohort $2 \mathrm{n}=14$ ) at total of twenty $(76.9 \%)$ completed the post-course survey. Among these, $85 \%$ identified as women and age ranged from 23 to 68 years $[\mathrm{M}=42.61(\mathrm{SD}=13.04)]$. Each class cohort was comprised of a heterogenous grouping of health professionals including physicians, learners (e.g., medical residents, fellows and students) as well as researchers. Notably, there were no differences (all p's $>$.05) between collected demographic characteristics between cohort $1(n=8)$ and cohort $2(n=12)$. All data analyses were conducted on the combined sample of twenty participants.

\section{Measures \\ Demographic Variables}

Due to the nature of this pilot program development project only two demographic variables (age, gender) were tracked for both cohorts.

\section{Primary Outcomes Workplace Well-Being}

The PERMAH workplace well-being profiler is a 23-item measure that assesses each domain of the PERMA well-being model [7] specifically as it relates to one's professional life/work [8]. The domains are: Positive emotion, Engagement, Positive relationships, Meaning and Accomplishment plus Perceived Health. Each item is presented with an 11-point (0-least to 10-most) likert-type format with anchors matching the stem content (e.g., Never Always, Not at all - Completely, Terrible - Excellent). The PERMA model measurement has been shown to be a valid format in which to measure the overall well-being construct. In this version of the PERMA(H) workplace profiler, additional items to assess Negative emotion (3-items) and Loneliness (1-item) are also included.

\section{Flourishing}

The Positive and Negative Affect Schedule [19] is a 20 -item self-report measure of positive and negative affect. Each item is a mood state adjective (e.g., "distressed" or "enthusiastic") and is rated on a scale of 1 ("very slightly or not at all") to 5 ("extremely") within a certain time instruction (i.e., moment, today, past few days, past few weeks, year, and general). The negative items are summed for the NA scale for negative affect, and the positive items are summed for the PA scale for positive affect. The PANAS has good internal consistency reliability across different time instructions for both the PA scale $(\alpha=0.86$ to $\alpha=0.90)$ and the NA scale $(\alpha=0.84$ to $\alpha=0.87)$. Test-retest reliability ranges from $r=0.47$ to $r=0.68$ for the PA scale and $r=0.39$ to $r=0.71$ for the NA scale. The flourishing ratio [10] is the calculated ratio of the positive PANAS total score divided by the negative PANAS total score. A ratio of $>3: 1$ positive-to-negative is regarded as "flourishing." Ratios of 1:1 are thought to be indicative of only moderate levels of mental health [16].

\section{Secondary Outcomes \\ Resilience}

The six items of the Brief Resilience Scale (BRS) [17] consists of three positively and three negatively worded items. Respondents are instructed to "indicate the extent to which you agree with each of the following statements by using the following scale: $1=$ strongly disagree, $2=$ disagree, $3=$ neutral, $4=$ agree, $5=$ strongly agree." The BRS score is computed by reverse scoring the three negatively coded items and subsequently calculating the mean of the six items.

\section{Mindfulness}

The 10-item Cognitive and Affective Mindfulness Scale - Revised (CAMS-R; 18) is a measure that broadly captures the construct of mindful approaches to thoughts and feelings. Participants respond to the item set using the following instructions: "People have a variety of ways of relating to their thoughts and feelings. For each of the items below, rate how much each of these ways applies to you." Each of the 10 items are rated on a 4-point likert scale from $1=$ "Rarely or not at all" to 4 = "Almost always" The CAMS-R has been determined to adequately sample the four domains of mindfulness (attention, present-focus, awareness, acceptance/non-judgment) but is most reliable with a single factor structure (i.e., one total score). The overall CAMS-R has demonstrated acceptable levels of internal consistency $(\alpha=.77)$ in other study samples.

\section{Self-Efficacy}

A total of six items assessed participants' self-efficacy to utilize concepts presented in each of the topic domains plus perceived ability to "serve as a local trainer/resource" for the course content. Respondents rated self-efficacy from 1 = "No ability" to 5 = "Expert."

\section{Well-Being Intervention: CU Flourish}

The CU Flourish curriculum was developed as an evidence-based intervention to assist in knowledge base and skill development to enhance workplace well-being and flourishing. The course was voluntary, free of charge and offered solely for the purposes of participants' self-interest and needs with no additional incentive offered by the Resilience Program. The approach integrates content and experiential exercises from positive psychology [7, 10] and values mindfulness-based behavioral interventions (e.g., Acceptance and Commitment Therapy, [9]). 


\begin{tabular}{llcccccccc}
\hline & \multicolumn{3}{c}{ Pre-Course } & \multicolumn{7}{c}{ Post-Course } \\
\hline Measure & M & SD & Alpha* & M & SD & Alpha* & t (19) & $p$ \\
\hline Total Well-Being Score 6.87 & 1.56 & 0.94 & 7.29 & 1.33 & 0.93 & 2.079 & .051 \\
Flourishing Ratio & 2.11 & 0.86 & -- & 2.44 & 0.87 & -- & 2.097 & .050 \\
Positive Affect & 33.35 & 7.27 & 0.90 & 34.62 & 6.25 & 0.92 & 1.066 & .300 \\
Negative Affect & 17.70 & 5.81 & 0.85 & 15.35 & 3.86 & 0.79 & 2.849 & .010 \\
Resilience & 3.44 & 0.62 & 0.81 & 3.56 & .737 & 0.87 & 1.240 & .231 \\
Mindfulness & 24.68 & 5.13 & 0.85 & 27.42 & 4.54 & 0.86 & 3.840 & .001 \\
\end{tabular}

NOTE: *Denotes Internal Consistency Cronbach's Alpha

An overview of course modules, content and exercises is presented in Table 1.

Although several modules of CU Flourish had been presented to various groups (e.g., medical residency programs) within the University of Colorado School of Medicine the content had not been presented in totality and in systematic fashion. Rather, members of the broader School of Medicine and health sciences campus were able to participate "a la carte" in modules. That is, each module was presented as a lunchtime brown bag and those interested could attend one or more of the topic modules. Similarly, various groups requested that one or more topics be presented outside of the course context or sequence. Overall, we found that attendance in this format was less than desired and the potential effect of the course content may not be realized in this format. The CU Flourish curriculum is currently a 5-module course. The modules are covered in 4,1-hour classes held one time each week.

\section{Data Analysis}

Among those who completed the post course surveys, missing data points were minimal with three missing data points. All of the missing data points were in the PERMAH Work Profiler (1 item Positive Emotion, 2 items Loneliness) scales. In each of these instances, the respective item mean was inserted in place of the missing data point. The Loneliness item is not included in PERMAH Work Profiler scale total scores. Therefore, for purposes of computation of scale scores and outcomes, only 1 item was missing. Among those who did not complete the majority of the post-course survey, one completed only the first measure and discontinued the survey. That participant's data was not included in final analyses. The remaining participants did not complete any portion of the survey. Given the exploratory pilot nature of this program development effort, values from the incomplete surveys were not imputed to facilitate "intent to treat" analyses. The completed data was analyzed descriptively and with univariate repeated measures analyses (i.e., paired t-tests). Again, given the exploratory pilot nature of the project, post-hoc adjustments (e.g., Bonferroni Correction) for multiple comparisons were not applied.

\section{RESULTS}

\section{Primary Outcomes}

Among our primary outcomes, at post course measurement, overall PERMAH Workplace Well-being scores increased ( $\mathrm{p}=.051)$. There were significant increases in the Flourishing Ratio scores from Pre- to Post-Course ( $p$ $=.05$ ). This result was largely influenced by a decrease in total Negative Affect scores on the PANAS $(p=.01)$. There was little change in PANAS Positive Affect scores (Table 2).

Notably, the change in overall Well-being scores was largely influenced by increases in the PERMAH domains of Positive Emotion ( $p=.05)$ and Meaning $(p=.05)$. Scores in all other PERMAH domains of Engagement, Positive Relationships, Accomplishment and Health also increased but did not reach significance. See Figure 1. Additional PERMAH items assessing Negative Emotion decreased from pre-course $[\mathrm{M}=3.79(\mathrm{SD}=2.22)]$ to post-course $[\mathrm{M}=3.67(\mathrm{SD}=1.95)]$ as did the single item assessing Loneliness at work [pre-course $\mathrm{M}=3.90$ $(\mathrm{SD}=3.09)$, post-course $\mathrm{M}=3.62(\mathrm{SD}=2.57)]$ but did not reach significance.

\section{Secondary Outcomes}

Among secondary outcome measures, the total Mindfulness (CAMS-R) scores increased significantly from pre- to post-course $(\mathrm{p}=.001)$. Total Resilience (BRS) scores increased slightly and not significantly. See Table 2.

Post-course Self-efficacy ratings among cohort 2 participants indicated that overall, participants felt confident in their ability to apply the skills presented in the course modules. Participants rated self-efficacy highest in their ability to cultivate positive emotion, use mindfulness techniques and apply the PERMAH model of well-being. The lowest rating was in perceived ability to serve as a local trainer or expert (see Table 3 on next page).

\section{Open-ended Comments}

Approximately one third of participants in each of the cohorts elected to write comments at the end of the postcourse survey. The comments had two primary themes: 1 ) expressing gratitude for the opportunity to take the course and 2) expressing a desire to be able to take additional courses or "deeper" coursework (e.g., one participant requested a "CU Flourish 2.0") to further develop skills. Other comments concerned logistics regarding day/time of classes and a desire to have weekly calendar reminders of next class. Anecdotally, during final class discussion in Cohort 1, participants felt that the 4 classes were not sufficient to allow for integration of 5 modules of content. Participants shared that they would attend a 5-week course in order to be able to more fully grasp and apply the skills and concepts presented in the curriculum. 


\begin{tabular}{llccccc}
\hline & \multicolumn{7}{l}{ Pre-Course } & & \\
\hline Measure & M & SD & M & SD & t (19) & $p$ \\
\hline Positive Emotion & 6.67 & 1.89 & 7.17 & 1.43 & 2.552 & .019 \\
Engagement & 6.33 & 1.91 & 6.82 & 1.68 & 1.777 & .092 \\
Relationships & 6.82 & 1.79 & 7.08 & 1.69 & 0.928 & .365 \\
Meaning & 7.48 & 1.83 & 8.03 & 1.46 & 2.342 & .030 \\
Accomplishment & 6.92 & 1.96 & 7.28 & 1.49 & 0.944 & .357 \\
Health & 7.17 & 1.95 & 6.85 & 1.65 & 1.224 & .236 \\
\hline
\end{tabular}

\section{DISCUSSION}

The present pilot study examined the effectiveness of CU Flourish in increasing workplace Well-being and Flourishing in a sample of health care professionals and learners. The sample was diverse, one comprised of many types of health care professionals. Although the demographic information collected in our groups was minimal, it is perhaps noteworthy that the age range in our cohorts was similarly broad spanning approximately four decades. There did not seem to be any pattern or trend for different age cohorts in whether or not the participants continued to participate and attend the classes. This is, in itself, encouraging with respect to the degree to which this seems to imply that participants find the course to be pertinent regardless of age or life/career stage.

Our first primary hypothesis was that there would be an increase in overall PERMAH workplace well-being scores. This hypothesis was supported with a p-value $(\mathrm{p}=.051)$. Although each of the six assessed PERMAH domain scores increased at post-course assessment, the increase in overall PERMAH well-being score was primarily due to increases in Positive Emotion and Meaning domains each of which were significant (See Figure 1). This finding was not surprising because the focus areas throughout the CU Flourish curriculum tend to "tap into" both of these domains. As shown in Table 1, we explicitly highlight modules with content related to these domains. We also note that although connection to these domains is explicit where these are noted, that other domains may be simultaneously (and serendipitously) enhanced. Seligman [7] and Butler and Kern [8] underscore that the domains are positively intercorrelated and that "improvement" in one may foster concurrent changes in others.

In the CU Flourish curriculum, mindfulness and present moment awareness concepts and practice are reinforced throughout the course as "core competencies" in enhancing well-being. Present moment awareness and the distinction between here-and-now presence and the past are introduced in module one. Mindfulness exercises are included each of the subsequent modules. It was, therefore, not surprising that pre- to post-course mindfulness scores increased $(p=.001)$.

One interpretation of our findings regarding these outcomes may be simply that the curriculum included content and exercises specifically targeting them. Ivtzan and colleagues [19] found results similar to the present study with increased scores in well-being, self-compassion, positive emotion, positive engagement with others (i.e., relationships) and engagement subsequent to an 8-week online positive mindfulness program. Another explanation may be that these observed effects are interrelated and possess shared mechanisms of change. Garland et al [14] propose a "mindfulness-to-meaning" theory in which they describe ways in which the practice of present moment nonjudgmental awareness actually enhances one's ability to generate more flexible ways of appraising adverse events. They pose that vis-avis mindfulness practice, a natural positive reappraisal occurs allowing for a "savor(ing) of the positive aspects of experience." They note that through "fostering positive reappraisals and emotions, mindfulness may generate deep eudaimonic meanings that promote resilience and engagement with a valued and purposeful life" [14].

The post-course self-efficacy ratings suggest that, to some degree, participants had confidence in their ability to continue to apply the content and employ skills from the curriculum (see Table 4 on next page). Among the highest of these ratings was to cultivate positive emotion and employ mindfulness techniques throughout their day. This finding, too, may be explained by directly related course content or as another example of the "mindfulness to meaning" theory [14].

\section{LIMITATIONS}

As with all research endeavors, study limitations should be accounted for when interpreting findings. This is especially true when considering pilot feasibility projects such as the present study. This study sample was comprised of two small convenience sub-samples of apparently highly motivated individuals who participated in the CU Flourish course without external incentive to do so. The sample size alone limits interpretation of our study outcomes. Additionally, a large proportion participants in each of the cohorts did not complete the post-course surveys. If baseline scores of measures were carried forward (i.e., intent-to-treat analysis), the encouraging outcomes we have reported herein might no longer be present. Similarly, a series of univariate analyses were conducted without correction for experiment wise effects and there was not a control comparison group. Finally, we collected very few demographic variables in the course offering which limit our ability to ascertain how useful (or appropriate) the CU Flourish curriculum may be in persons who have diverse characteristics and backgrounds. 


\begin{tabular}{lcc}
\hline Item & M & SD \\
\hline Use the PERMAH Model of Well-Being to assess your personal well-being. & 3.82 & 1.17 \\
Use mindfulness techniques throughout your day & 3.91 & 0.70 \\
Use the positivity "Flourishing Ratio" as a way to understand your emotional & 3.54 & 0.82 \\
experiences & & \\
Identify opportunities to cultivate positive emotion & 4.20 & 0.63 \\
Use values Choice Point and clarification techniques to make values-based decisions & 3.64 & 1.21 \\
Apply Grit concepts to my daily activities & 3.73 & 1.00 \\
Serve as a local trainer/resource for the CU Flourish course content & 2.81 & 0.87 \\
\hline Note. Likert scale ratings 1 = Not at all to 5 = Expert; Cohort 2 participants only (n=11) & &
\end{tabular}

\section{Future Directions}

Further evaluation of the CU Flourish curriculum is either planned or currently underway. First, CU Flourish is being offered to different and larger groups of recipients including intact teams and a variety of learners including medical residents and fellows. Second, the feasibility of different modalities of delivery is being evaluated in a planned wait list control trial via distance learning technology. This, in addition to training trainers for in-person delivery, would allow for broad scaling of the curriculum for potential impact across a wide audience. As such fifteen participants ( 12 physicians, two psychologists and one hospital chaplain) recently completed a three-day CU Flourish Train-the-Trainer course and those results are reported elsewhere [20]. Third, dissemination of CU Flourish more broadly to diverse groups of participants is planned. Finally, a closer examination of refined curriculum content and mechanisms of action is the penultimate goal of the creators of this curriculum.

\section{CONCLUSION}

Taken together, the findings of this pilot study suggest that CU Flourish is a feasible and potentially effective curriculum to enhance participant Well-being and Flourishing among health care professionals and learners. The curriculum meets all development goals originally set forth. As it stands, CU Flourish is a brief and evidence-based curriculum embedded within an empirically derived framework - the PERMAH model of well-being $[7,8,21]$. It appears that, based on initial findings, participants of all ages find the content to be personally applicable and useful. Further evaluation of the effectiveness of curriculum content and modes of delivery are underway.

\section{REFERENCES}

1. Dyrbye LN, Massie FS Jr, Eacker A, Harper W, Power D, Durning SJ, et al. Relationship between burnout and professional conduct and attitudes among US medical students. JAMA. 2010 Sep;304(11):1173-80.

2. Shanafelt T, Goh J, Sinsky C. The Business Case for Investing in Physician Well-being. JAMA Internal Medicine Clinical Review and Education. 2017; E1-E7. https://doi.org/10.1001/ jamainternmed.2017.4340.

3. Moss M, Good VS, Gozal D, Kleinpell R, Sessler $\mathrm{CN}$. An official critical care societies collaborative statement: Burnout syndrome in critical care health professionals: A call for action. Jnl Critical Care. 2016: 25 (4) Available from: doi:https://doi. org/10.1080/17439760.2017.138843.

4. Sackett DL, Straus SE, Richardson WS, Rosenberg W, Haynes RB. (2000). Evidence based medicine: How to practice and teach EBM (2nd ed.). Schrijver I. Pathology in the medical profession? Taking the pulse of physician wellness and burnout. Arch Pathology Lab Med. 2016; 140: 976-982.

5. Slavin SJ, Schindler D, Chibnall JT, Fendell G, Shoss M. PERMA: a model for institutional leadership and culture change. Acad Med. 2012 Nov;87(11):1481.

6. Eckleberry-Hunt J, Van Dyke A, Lick D. Tucciarone J. Changing the Conversation From Burnout to Wellness: Physician Wellbeing in Residency Training Programs. Jnl Grad Med Educ; 2009. pp. 225-30.

7. Seligman M. Flourish: A visionary new understanding of happiness and well-being. New York (NY): Free Press; 2011.

8. Butler J, Kern ML. The PERMA-Profiler: A brief multidimensional measure of flourishing. Intl Jnl Wellbeing. 2016; 6(3): 1-48. Available from: doi:https://doi.org/10.5502/ijw.v6i3.526.

9. Bohlmeijer ET, Fledderus M, Rokx TA, Pieterse ME. Efficacy of an early intervention based on acceptance and commitment therapy for adults with depressive symptomatology: evaluation in a randomized controlled trial. Behav Res Ther. 2011 Jan;49(1):62-7.

10. Kashdan TB, Gallagher MW, Silvia PJ, Winterstein BP, Breen WE, Terhar D, et al. The curiosity and exploration inventory-II: Development, factor structure, and psychometrics. J Res Pers. 2009 Dec;43(6):987-98.

11. Institute of Medicine. Crossing the quality chasm: A new health system for the 21st century. Washington (DC): National Academy Press; 2001.

12. Sackett DL, Straus SE, Richardson WS, Rosenberg W, Haynes RB. Evidence based medicine: How to practice and teach EBM. 2nd ed. London: Churchill Livingstone; 2000. 
13. Bolier L, Haverman M, Westerhof G, Riper H, Smit F. Bohlmeijer E. Positive psychology interventions: A meta-analysis of randomized controlled studies. BMC Public Health. 2013; 13: 119. Available from: doi: https://doi.org/10.1186/1471-2458-13-119..

14. Garland EL, Farb NA, Goldin P, Fredrickson BL. Mindfulness Broadens Awareness and Builds Eudaimonic Meaning at the Attention-Appraisal-Emotion Interface: A Process Model of Mindful Positive Emotion Regulation. Psychol Inq. 2015;26(4):293-314.

15. Harris R. Choice Point 2.0, [Internet] 2017. Available from: http//actmindfully.com.au

16. Fredrickson BL. Updated thinking on positivity ratios. Am Psychol. 2013 Dec;68(9):814-22.

17. Smith BW, Dalen J, Wiggins K, Tooley E, Christopher P, Bernard J. The Brief Resilience Scale: Assessing the Ability to Bounce Back. Intl Jnl Behav Med. 2008; 15: 194-200. Available from: doi: https://doi.org/10.1080/10705500802222972.

18. Feldman G, Hayes A, Kumar S, Greeson J, Laurenceau JP. Mindfulness and emotion regulation: The development and initial validation of the
Cognitive and Affective Mindfulness Scale Revised (CAMS-R). J Psychopathol Behav Assess. 2007;29(3):177-90.

19. Watson D, Clark LA, Tellegen A. Development and validation of brief measures of positive and negative affect: the PANAS scales. J Pers Soc Psychol. 1988 Jun;54(6):1063-70.

20. Beacham A, Reese J. Training trainers to delivery well-being curriculum to healthcare providers: A pilot study. Unpublished manuscript, n.d.

21. Goodman F, Disabato D, Kashdan T, Kauffman S. Measuring well-being: A comparison of subjective well-being and PERMA. J Posit Psychol. 2017:1-12.

22. Lundgren T, Luoma J, Dahl J, Strosahl K, Melin L. The Bull's-Eye Values Survey: A Psychometric Evaluation. Cognit Behav Pract. 2012;19(4):518-26.

23. Duckworth A. Grit: The Power of Passion and Perseverance. New York: Scribner; 2016.

24. Dweck C. What having a growth mindset actually means. Harv Bus Rev. 2016;(January):2-4. 